\title{
Evolução e viabilização de um centro cirúrgico ambulatorial para cirurgias de catarata em larga escala em um hospital universitário
}

\author{
Evolution and viability of an outpatient surgery center for cataract surgery \\ on a large scale in a university hospital
}

Newton Kara-Junior ${ }^{1}$, Rodrigo França de Espíndola ${ }^{1}$

\section{RESUMO}

Objetivo: Analisar a evolução do número de cirurgias realizadas no centro cirúrgico ambulatorial de um hospital universitário e avaliar sua viabilidade financeira durante e após a interrupção da Campanha Nacional de Catarata em 2006.

Métodos: Foi realizado um estudo analítico retrospectivo entre 2005 e 2009 no Hospital das Clínicas da Faculdade de Medicina da Universidade de São Paulo (HC-FMUSP) em que foram avaliados a viabilidade econômica do centro cirúrgico ambulatorial, o número de cirurgias de catarata realizados e o número de cirurgiões presentes diariamente naquela unidade.

Resultados: Seria necessária a realização de pelo menos 400 procedimentos mensais para garantir a viabilidade financeira do centro cirúrgico ambulatorial. Este número ficou abaixo do esperado nos anos de 2008 e 2009 (média de 370,6 e 390,1 cirurgias respectivamente). O número de estagiários de catarata diminuiu de 13 em 2005 para 3 em 2009.

Conclusão: $O$ principal fator para a redução no número de cirurgias de catarata realizadas no centro cirúrgico ambulatorial após 2006 foi a dificuldade de acesso da população necessitada ao hospital, decorrente de restrições à realização de projetos de triagem. A maior utilização das salas cirúrgicas por outras clínicas e a diminuição na admissão de novos cirurgiões, adequaram e viabilizaram o centro cirúrgico ambulatorial para a nova realidade políticoeconômica.

Descritores: Extração de catarata; Otimização; Facoemulsificação/economia; Hospitais de ensino; Cegueira/prevenção e controle

\begin{abstract}
Purpose: To analyze the number of surgeries performed in outpatient surgical center at a university hospital and to assess its financial viability during and after the interruption of the Cataract National Campaign in 2006.

Methods: Retrospective analytical study between 2005 and 2009 at the Clinical Hospital of the University of São Paulo (HC-FMUSP) which evaluated the economic viability of the outpatient surgical center, the number of cataract surgeries performed and the number of surgeons present daily in that unit.

Results: It would be necessary to perform at least 400 procedures monthly to ensure the financial viability of the outpatient surgical center. This number was lower than the expected in the years of 2008 and 2009 (average of 370.6 and 390.1 surgeries respectively). The number of cataract fellows decreased from 13 in 2005 to 3 in 2009.

Conclusion: The main factor for the reduction in the number of cataract surgeries performed in the outpatient surgical center after 2006 was the difficulty of access of the population to the hospital due to restrictions on the development of screening projects. The increased use of the operating rooms by other clinics and the decrease in the admission of new surgeons, made the outpatient surgical center appropriate and viable for the new political-economic reality.
\end{abstract}

Keywords: Cataractextraction; Optimization; Phacoemulsification/economics; Hospital, teaching; Blindness/prevention \& control

\section{INTRODUÇÃO}

A catarata é considerada a principal causa de cegueira, sendo responsável por cerca da metade do número de cegos no mundo, cerca de 20 milhões de pessoas. Devido ao aumento da população idosa, estima-se que a prevalência da cegueira por catarata duplique até o ano de $2010^{(1)}$.

A cirurgia da catarata apresenta alta eficiência, favorável custo-benefício no tratamento da doença e na reabilitação visual do indivíduo e oferece grande impacto para a sociedade(2-5).

Em 1998, por iniciativa do Conselho Brasileiro de Oftalmologia (CBO), do Governo Federal (Ministério da Saúde) e com o auxílio das escolas de medicina, foi instituída a Campanha Na-

\footnotetext{
Trabalho realizado no Setor de Catarata, Departamento de Oftalmologia, Hospital das Clínicas, Faculdade de Medicina, Universidade de São Paulo - USP - São Paulo (SP), Brasil. ${ }^{1}$ Médico, Setor de Catarata, Hospital das Clinicas, Faculdade de Medicina, Universidade de São Paulo - USP - São Paulo (SP), Brasil.

Endereço de correspondência: Rodrigo França de Espíndola. Praça das Hortências, 70 - Cond. Portal de Itu - Itu (SP) - CEP 13301-689 - E-mail: rodrigo166@uol.com.br Recebido para publicação em 17.06.2010

Aprovado em 05.11.2010
}

cional de Catarata (CNC). O acesso ao tratamento foi facilitado com a realização de Projetos-Catarata em todo o país e o Governo Federal determinou que o financiamento das cirurgias pelo SUS fosse em forma de "extra-teto" para as instituições credenciadas, garantindo o financiamento de todas as cirurgias realizadas ${ }^{(4,6-7)}$.

Com o intuito de colaborar com a política nacional e a necessidade social de diminuir a prevalência da cegueira por catarata, além do interesse em melhorar as condições de ensino da cirurgia, foi criado, em um hospital universitário, em 1999, um centro cirúrgico ambulatorial (CCA). Esta unidade era especializada em cirurgias de catarata por facoemulsificação em larga escala, oferecendo à população necessitada tratamento com os melhores insumos disponíveis e oportunidade de treinamento supervisionado e de qualidade para novos cirurgiões. Em 10 anos de funcionamento, 201 oftalmologistas foram treinados no CCA para realizar cirurgias de catarata $^{(8)}$.

Contudo, em 2006 o Governo Federal alegando falta de verba, descontinuou o repasse "extra-teto" e desestimulou a realização dos Projetos Catarata(4)

O objetivo deste estudo é analisar a evolução do número de cirurgias realizadas no CCA e avaliar sua viabilidade financeira, durante e após a mudança da política governamental. 


\section{MÉTODOS}

Foi realizado estudo clínico analítico retrospectivo no Hospital das Clínicas da Faculdade de Medicina da Universidade de São Paulo (HC-FMUSP).

O CCA possui área útil de 250,71 m², com 5 salas cirúrgicas em funcionamento 5 dias por semana, 12 horas por dia, sendo que a primeira e a última hora de funcionamento são reservadas, respectivamente, para admissão e preparo do paciente, e para limpeza do local. Portanto, o período disponível para a realização das cirurgias é de 10 horas.

Os equipamentos e insumos utilizados rotineiramente para cirurgia de catarata no CCA são: 4 microscópios cirúrgicos (Zeiss III, Carl Zeiss), 1 microscópio cirúrgico (Opto, São Carlos), 2 facoemulsificadores Infiniti (Alcon Labs, Fortwoth, TX), 2 facoemulsificadores Legacy (Alcon Labs, Fortwoth, TX) 2 autoclaves (Statin), "pacote cirúrgico" pré-montado contendo todos os insumos necessários e descartáveis (Alcon Custom Pack, Alcon Labs, Fortwoth, TX), lente intraocular acrílica dobrável (Acrysof Type 7: Alcon Labs, Fortwoth, TX) para facoemulsificação e viscoelástico dispersivo (Viscoat - Alcon Labs, Fortwoth, TX).

Quanto aos recursos humanos, estão presentes no CCA 42 residentes do Serviço: residentes de primeiro (R1), segundo (R2) e terceiro anos (R3); além dos residentes estrangeiros, que são admitidos anualmente para cumprir um programa de estágio com duração de três anos. Todos os residentes participam uma vez por semana das atividades do CCA. Há também a participação de médicos preceptores; ex-residentes; estagiários do Setor de Catarata e os coordenadores de cada dia cirúrgico.

As variáveis analisadas foram: quantidade mínima de cirurgias (facoemulsificação) necessária para garantir a viabilidade econômica do CCA; número de cirurgias realizadas no CCA; e número de cirurgiões presentes diariamente no CCA.

\section{RESULTADOS}

\section{Avaliação da viabilidade econômica do CCA}

Estudos anteriores analisaram gastos com recursos humanos e materiais, e inferiram que o custo fixo de uma sala cirúrgica do CCA por dia foi de $\mathrm{R} \$ 588,13^{(8-9)}$. Avaliando que era possível realizar 16 cirurgias por dia (facoemulsificação) em uma sala do CCA, foi estimado o custo fixo relativo por cirurgia de $R \$ 36,75$. Assim, considerando o custo variável de cada cirurgia de $\mathrm{R} \$ 468,63$ e o reembolso do Sistema Único de Saúde (SUS) para cada procedimento de R\$ 643,00, na situação de otimização máxima do CCA, o lucro para o hospital com cada cirurgia era de $\mathrm{R} \$ 137,62^{(8)}$.

Neste contexto, ao reduzir a eficiência do CCA realizandose 8 cirurgias por sala, o custo fixo por cirurgia passaria a ser de $\mathrm{R} \$ 73,51$ e o lucro do hospital seria de $\mathrm{R} \$ 100,87$ por procedimento. No caso da realização de 4 cirurgias por sala, o custo fixo seria de $\mathrm{R} \$ 147,03$, o que reduziria o lucro do hospital para $\mathrm{R} \$ 27,33$. Desta forma, infere-se que "4" seria o número mínimo de cirurgias por sala, que propiciaria lucro ao hospital e, consequentemente, viabilidade financeira.

Baseado nas características físicas, nos recursos humanos e materiais do CCA, bem como no custo financeiro das cirurgias, e considerando que as 5 salas cirúrgicas fossem destinadas à facoemulsificação, seria necessária a realização de pelo menos 400 cirurgias mensais para garantir a viabilidade financeira do CCA.

\section{Número de Cirurgias anUalmente Realizadas no CCA}

O número de cirurgias realizadas anualmente no CCA a partir de 2005 está demonstrado na tabela 1.

\section{NúmERo de CIRURGIÕES PRESENTES DIARIAMENTE NO CCA}

Estão diariamente presentes no CCA: 3 residentes de cada ano (R1, R2 e R3), um preceptor ou ex-residente, o coordenador do dia cirúrgico, além de um número variável de estagiários do Setor de Catarata.

O número de estagiários que ingressaram no Setor de Catarata, permanecendo por pelo menos dois anos na Clínica, variou anualmente em decorrência da necessidade de atendimento à demanda cirúrgica do Serviço (Tabela 2). Cada estagiário opera uma vez por semana no CCA.

A tabela 3 mostra o número de oftalmologistas presentes diariamente no CCA.

\section{DISCUSSÃO}

Calcula-se que foram realizadas em média, entre 1996 e 1998, 132 mil cirurgias de catarata por ano, financiadas pelo SUS. Considerando-se que o Brasil necessitaria de 570.000 cirurgias de catarata por ano para não aumentar o número de cegos, nota-se que aquela proporção é insuficiente até mesmo para compensar novos casos surgidos no período, o que aumenta a prevalência de deficiência visual e cegueira(4).

Em 1998 a CNC mobilizando mais de 3.500 oftalmologistas, realizou nos 3 anos subsequentes uma média anual de 263 mil cirurgias de catarata, praticamente o dobro dos anos anteriores, quantidade esta que foi aumentando até atingir em 2005, a marca de 311 mil cirurgias. Para que esse número adicional de cirurgias fosse possível, bastou o Projeto-Catarata facilitar em todo o país o acesso ao tratamento. Ao Governo

\section{Tabela 1. Distribuição do número de cirurgias realizadas anualmente no centro cirúrgico ambulatorial (CCA) a partir do ano de 2005}

\begin{tabular}{lc}
\hline Ano & Número de cirurgias (média mensal) \\
\hline 2005 & $5.078(423,2)$ \\
2006 & $4.950(412,5)$ \\
2007 & $6.092(507,7)$ \\
2008 & $4.447(370,6)$ \\
2009 & $4.668(390,1)$ \\
\hline
\end{tabular}

Tabela 2. Número de estagiários que ingressaram anualmente no Setor de Catarata

\begin{tabular}{lc}
\hline Ano & Número de estagiários \\
\hline 2005 & 4 \\
2006 & 13 \\
2007 & 19 \\
2008 & 17 \\
2009 & 3 \\
\hline
\end{tabular}

Tabela 3. Distribuição do número de oftalmologistas presentes diariamente no centro cirúrgico ambulatorial

\begin{tabular}{lc}
\hline Ano & Número de oftalmologistas \\
\hline 2006 & 14 \\
2007 & 17 \\
2008 & 18 \\
2009 & 15 \\
\hline
\end{tabular}


Federal, coube permitir que o financiamento das cirurgias pelo SUS fosse em forma de "extra-teto" para as instituições credenciadas $^{(4-6)}$.

O Serviço de Oftalmologia do Hospital das Clínicas da Universidade de São Paulo, por exemplo, elevou o número de médicos residentes de 5 para 14 ao ano e aumentou o número de cirurgias de catarata realizadas anualmente de $836 \mathrm{em}$ 1998, para 5.078 em 2005. Estimulou a produtividade dos médicos e do pessoal auxiliar envolvido no atendimento, otimizou o centro cirúrgico e facilitou o acesso da população necessitada ao hospital por meio de mutirões continuados (Projetos-Catarata) $)^{(9)}$

Em 2006, o Ministério da Saúde descontinuou a CNC sob alegação de falta de verbas para custear o número adicional de cirurgias ("extra-teto"). Os hospitais voltaram a ter cotas limitadas para a realização dos procedimentos cirúrgicos.

Essa medida causou impacto negativo na progressão do número de cirurgias realizadas anualmente no país. Em 2006 foram computadas somente 171.831 facectomias realizadas pelo SUS ${ }^{(4)}$, reduzindo assim, a utilização da capacidade instalada para cirurgia de catarata nos hospitais públicos e voltando a impor mais uma barreira ao acesso da população necessitada à cirurgia corretiva: a limitação da oferta cirúrgica.

Economia em saúde pública não se faz gastando menos, mas gastando-se melhor, e as vantagens socioeconômicas para - governo e para a sociedade da cirurgia de catarata por facoemulsificação já foram evidenciadas em outros estudos ${ }^{(5,10)}$. Assim, acredita-se que a priorização do financiamento federal para cirurgia de catarata pelo SUS seja mais uma opção política do que técnica.

Embora, no plano nacional a quantidade de facectomias realizadas pelo SUS tenha diminuído após o fim da $\mathrm{CNC}^{(4)}$, observou-se que o número de cirurgias de catarata realizadas pelo CCA nos dois anos seguintes ao encerramento da campanha pouco variou (Tabela 1). O motivo foi o sucesso de parcerias firmadas pelo hospital universitário com prefeituras de cidades vizinhas e organizações não governamentais para financiamento de cirurgias. Contudo, foi em 2008 com a proibição da realização de mutirões para triagem e captação de portadores de catarata nas dependências do hospital, que o acesso da população necessitada ao tratamento foi dificultado, e o número de cirurgias realizadas diminuiu drasticamente (Tabela 1).

Na época de vigência da CNC, as 5 salas do CCA eram utilizadas para cirurgia de catarata na maior parte do tempo. A partir de 2008, para evitar prejuízo econômico ao hospital, as facectomias foram concentradas em duas salas, e as demais utilizadas por outras clínicas cirúrgicas.
Também para adequar-se ao novo cenário em 2009, o número de estagiários que ingressaram no Setor de Catarata foi reduzido de 17 para 3, diminuindo de 18 para 15 o número de oftalmologistas diariamente presentes no CCA (Tabelas 2 e 3) e, consequentemente, reduzindo a atuação na formação de recursos humanos.

Nas condições propostas para este estudo, conclui-se que, o principal fator para a redução no número de cirurgias de catarata realizadas no CCA da instituição em questão, após a suspensão da CNC, foi a dificuldade de acesso da população necessitada ao hospital, decorrente de restrições à realização de projetos de triagem. A maior utilização das salas cirúrgicas por outras clínicas e a diminuição na admissão de novos cirurgiões, adequaram e viabilizaram o CCA para a nova realidade político-econômica.

Porém, é possível que estas soluções apresentadas para sustentabilidade financeira da capacidade cirúrgica instalada não tenham sido exequíveis em outras instituições que, estimuladas pelos incentivos da CNC, tenham ampliado sua estrutura física e de recursos humanos, resultando em possível prejuízo financeiro, o que poderia dificultar a aceitação de futuras ações de incentivo à cirurgia de catarata por parte dos gestores hospitalares.

\section{REFERÊNCIAS}

1. Leaming DV. Practice styles and preferences of ASCRS members-1998 survey. J Cataract Refract Surg. 1999;25(6):851-9.

2. Gillies M, Brian G, La Nauze J, Le Mesurier R, Moran D, Taylor H, Ruit S. Modern surgery for global cataract blindness: preliminary considerations. Arch Ophthalmol. 1998;116(1):90-2. Review.

3. Singh AJ, Garner P, Floyd K. Cost-effectiveness of public-funded options for cataract surgery in Mysore, India. Lancet. 2000;355(9199):180-4.

4. Kara-José Junior N, Cursino M, Whitaker E. Cirurgia de catarata: otimização de centro cirúrgico com utilização de pacote cirúrgico pré-montado. Arq Bras Oftalmol. 2004:67(2):305-9.

5. Kara-Junior N, Arieta CEL. Catarata senil. In: Kara-José N, Almeida GV, editores. Senilidade ocular. São Paulo: Roca; 2001. p.99-107

6. Kara-Jose N, Temporini ER. Catarata e cegueira: epidemiologia e prevenção. In: Arieta CE, editor. Cristalino e catarata. Rio de Janeiro: Cultura Médica; 2002. p.49-56.

7. Kara-José N, Bicas HEA, Carvalho RS. Cirurgia de catarata: necessidade social. São Paulo: C\&D - Editora e Gráfica; 2008. p.45-6.

8. Kara-Junior N, de Santhiago MR, Kawakami A, Carricondo P, Hida WT. Mini-rhexis for white intumescent cataracts. Clinics (São Paulo). 2009:64(4):309-12.

9. Kara-José Junior N, Avakian A, Lower LMT, Rocha AM, Cursino M, Alves MR. Facoemulsificação versus extração manual do cristalino: análise de custos. Arq Bras Oftalmol. 2004:67(3):481-9.

10. Sirtoli MG, Santhiago MR, Parede TR, de Espíndola RF, Carvalho RS, Kara-Jr N. Phacoemulsification versus extracapsular extraction: governmental costs. Clinics (São Paulo). 2010;65(4):357-61.

Ao enviar um artigo para publicacão, leia ATENTAMENTE as instrucões para autores, constante no final de cada fascículo. 